\title{
Matter-Wave Interferometry with Phase Fluctuating Bose-Einstein Condensates
}

\author{
G.-B. Jo, J.-H. Choi, C. A. Christensen, Y.-R. Lee, T. A. Pasquini, W. Ketterle, and D. E. Pritchard* \\ MIT-Harvard Center for Ultracold Atoms, Research Laboratory of Electronics, Department of Physics, \\ Massachusetts Institute of Technology, Cambridge, Massachusetts 02139, USA
}

(Received 26 June 2007; published 14 December 2007)

\begin{abstract}
Elongated Bose-Einstein condensates (BECs) exhibit strong spatial phase fluctuations even well below the BEC transition temperature. We demonstrate that atom interferometers using such condensates are robust against phase fluctuations; i.e., the relative phase of the split condensate is reproducible despite axial phase fluctuations. However, larger phase fluctuations limit the coherence time, especially in the presence of some asymmetries in the two wells of the interferometer.
\end{abstract}

PACS numbers: 03.75.Dg, 03.75.Lm, 39.20.+q

A noninteracting zero temperature Bose-Einstein condensate is the matter-wave analogue to the optical laser, and therefore the ideal atom source for atom interferometry. Finite temperature and atomic interactions profoundly change the coherence properties of a condensate and introduce phase fluctuations and phase diffusion. Those phenomena are of fundamental interest [1-6], but also of practical importance because they may limit the performance of atom interferometers [7-9]. This applies, in particular, to magnetic microtraps and waveguides (e.g., atom chips) [10] since tight confinement and elongated geometry enhances phase diffusion and phase fluctuations.

Phase diffusion is a quantum effect associated with the coherent splitting of the condensate. Number fluctuations lead to density fluctuations, which, due to interactions, cause fluctuations of the energy and cause diffusion of the relative phase proportional to the chemical potential times $\Delta N / N$, the amount of fluctuations in the relative atom number. In our previous work $[11,12]$, we showed that such phase diffusion could be dramatically reduced by number squeezing, increasing the coherence time. In this paper, we characterize and discuss the role of spatial phase fluctuations in an atom interferometer.

Phase fluctuations cause the condensate to break up into several quasicondensates with random phase; i.e., long range coherence is lost. This usually happens in elongated geometries when the temperature is sufficiently high to excite such modes [1,2], or in interacting one-dimensional condensates even at zero temperature due to quantum fluctuations [13]. Spatial phase fluctuations have two major consequences for atom interferometry. First, they speed up phase diffusion, since $\Delta N / N$ refers now to the atom number in a single quasicondensate. Second, they make the atom interferometer much more sensitive to random relative displacements of the split condensates, which have to be smaller than the coherence length, which, for condensates with phase fluctuations, can be much smaller than the size of the condensate.

A typical elongated trap geometry, realized by an atom chip, has an aspect ratio of $\sim 200[8,11,14]$, sufficient to induce phase fluctuations in a quasicondensate along the axial direction [1] already at very low temperatures (or in the $1 \mathrm{D}$ case, even at zero temperature). When the temperature of a condensate is above the characteristic temperature, $T^{*}=15 N\left(\hbar \omega_{z}\right)^{2} / 32 \mu$, where $\mu$ is the chemical potential, $N$ total atom number, $\omega_{z}$ axial trap frequency, and $\hbar$ the Planck's constant divided by $2 \pi$ [1], then thermal excitations of low energy axial modes lead to longitudinal phase fluctuations. For temperatures above $T^{*}$, the coherence length $L^{*}$ of a phase-fluctuating condensate is shorter than the length $L$ of the condensate $L^{*} / L=T^{*} / T$ [1].

Previous experiments $[8,11,14]$ on atom interferometry have operated in a regime where phase fluctuations are predicted to be present. However, their presence has not been observed because the interferometer was read out by integrating the interference fringes along the axial direction. Other experiments characterized phase fluctuations by interferometric techniques [15] and Bragg spectroscopy [16], but did not study the effect of phase fluctuations on an atom interferometer.

In this Letter we observe the axial phase fluctuations spatially resolved and characterize their effect on the coherence time of the atom interferometer. We show explicitly that atom interferometry can be performed in the presence of phase fluctuations. This has been expected [13], since for sufficiently short times after splitting, those fluctuations are identical for both condensates and therefore do not affect the measurement of the relative phase. For the same reason, atom interferometry is possible with thermal clouds of atoms [17]. However, already at short times, phase fluctuations degrade the contrast and can limit the coherence time. As we discuss below, we believe that this degradation is not due to the quantum effect of the increased relative number fluctuations in each quasicondensate because of the high degree of number squeezing, but is rather caused by asymmetries in the double-well potential leading to relative motion of the condensates.

Bose-Einstein condensates of $\sim 4 \times 10^{5}{ }^{23} \mathrm{Na}$ atoms in the $\left|F=1, m_{F}=-1\right\rangle$ state were transferred into a magnetic trap generated by the trapping wire on an atom chip and external bias field [14]. Using adiabatic rf-induced splitting $[8,18]$, a double-well potential in the vertical plane (paral- 
lel to the gravity direction) was formed as illustrated in Fig. 1(a) [19]. Gravity was compensated by a magnetic field gradient from the trapping wire. Typically, the separation of the two wells was $d \sim 6 \mu \mathrm{m}$, the height of the trap barrier was $U \sim h \times 10 \mathrm{kHz}$, and the difference of the trap bottom between two wells $\sim h \times 300 \mathrm{~Hz}$. The trapping frequencies were $\sim 2 \mathrm{kHz}$ (radial) and $\sim 10 \mathrm{~Hz}$ (axial). The absorption imaging light for data acquisition was resonant with the $|F=2\rangle \rightarrow\left|F^{\prime}=3\right\rangle$ cycling transition for the trapped atoms and was aligned perpendicular to the condensate axis [side imaging in Fig. 1(c)]. The atoms were optically pumped into the $|F=2\rangle$ hyperfine level with a pulse resonant with the $|F=1\rangle \rightarrow\left|F^{\prime}=2\right\rangle$ transition.

First, we characterized the presence of phase fluctuations in the condensate before splitting by observing density modulations of the expanded atomic cloud after $7 \mathrm{~ms}$ time of flight (Fig. 2 inset). In trap, the mean-field interaction energy suppresses density fluctuations, but ballistic expansion converts phase fluctuations into density modulations [2] since the initial velocity field is proportional to the gradient of the phase. The number of observed density striations of around ten is consistent with the ratio of the measured temperature of $\sim 650 \pm 100 \mathrm{nK}$ and the calculated value of $T^{*} \simeq 60 \mathrm{nK}$. Since the barrier height is comparable to the temperature, we assume that both condensates interact with the same heat bath. However, we do not expect any difference to the case of two separated thermal clouds.

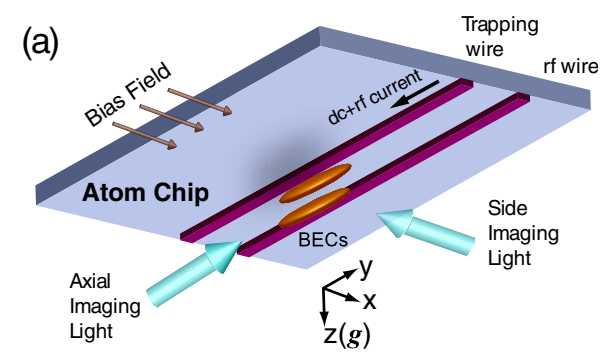

(b)

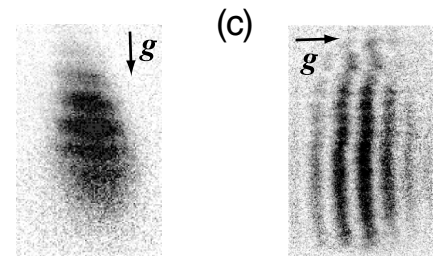

FIG. 1 (color online). Geometry of the atom chip interferometer. (a) Atoms were confined radially by the combined magnetic potential of a current-carrying wire and an external bias field. A pair of end cap wires (not shown) provided axial confinement. The single well was deformed into a vertical double well within $15 \mathrm{~ms}$ by adding rf current into the trapping wire dressing the atoms with oscillating rf fields. Absorption image was taken by a probe beam directed along the condensate axis [(b), axial imaging] and perpendicular to the condensate axis [(c), side imaging]. All data in this Letter were obtained using side imaging. The fields of view are $160 \times 260 \mu \mathrm{m}$ and $180 \times 100 \mu \mathrm{m}$ for axial and side imaging, respectively.
The longitudinal phase fluctuations were quantified by measuring the root-mean-square average of the density fluctuations as described in Fig. 2 [20]. The amount of phase fluctuations was controlled by changing the atom number and the temperature with rf-evaporation. The rf field generated by the rf wire [Fig. 1(a)] was swept down from $\sim 20 \mathrm{kHz}$ above the Larmor frequency at the trap center to a variable final value, leading to a variable chemical potential and temperature of the condensate (Fig. 2 inset) [21]. The variation of the spatial phase fluctuations with chemical potential is shown in Fig. 2.

Having firmly established the presence of phase fluctuations, we can now demonstrate the robustness of an atom interferometer against longitudinal phase fluctuations. For this, we split the condensates and observe the reproducibility of ten interference fringes obtained by recombining the condensates during ballistic expansion. The regular, almost straight interference fringes (Figs. 1 and 3) show that the spatial phase fluctuations are common mode and do not affect the relative phase in a major way.

However, when we increase the amount of phase fluctuations, we observe an increasing blurring or waviness of the interference fringes (Fig. 3). The number of wiggles of the waviness is comparable to the modulation pattern observed in the ballistic expansion of single condensates (Fig. 2). Of course, without any technical imperfection in

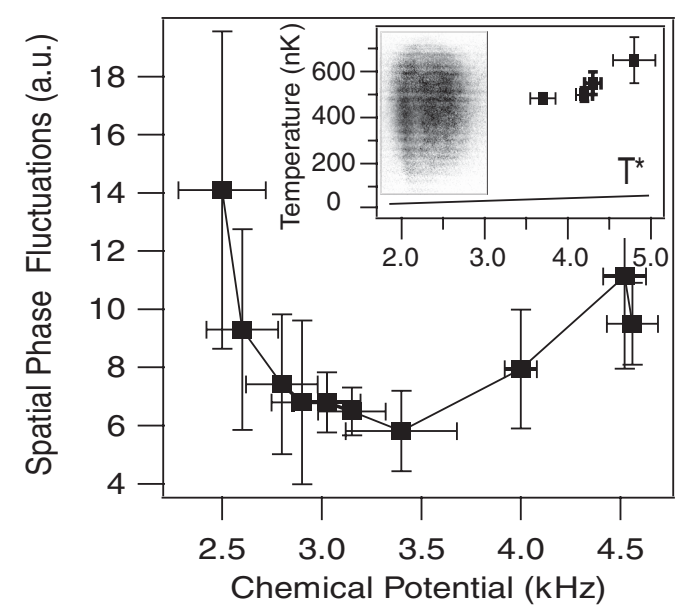

FIG. 2. Spatial phase fluctuations in a single condensate. The phase fluctuations were characterized by observing the density modulations in an absorption image of the expanded cloud after $8 \mathrm{~ms}$ time-of-flight (see inset) and calculating the rms fluctuations as described in Ref. [2]. The chemical potential (or atom number) was controlled by additional rf-evaporative cooling. The temperature of the condensate is shown in the inset graph. For chemical potentials less than $3.5 \mathrm{kHz}$, we could not measure the temperature of a condensate due to the lack of discernible thermal atoms. The observed phase fluctuations do not decrease monotonically, but show a minimum at the chemical potential of $\sim 3.5 \mathrm{kHz}$, probably because the effect of the lower temperature was more than offset by the loss in the atom number. In the inset graph, $T^{*}$ displays the characteristic temperature for the onset of the phase fluctuations. 


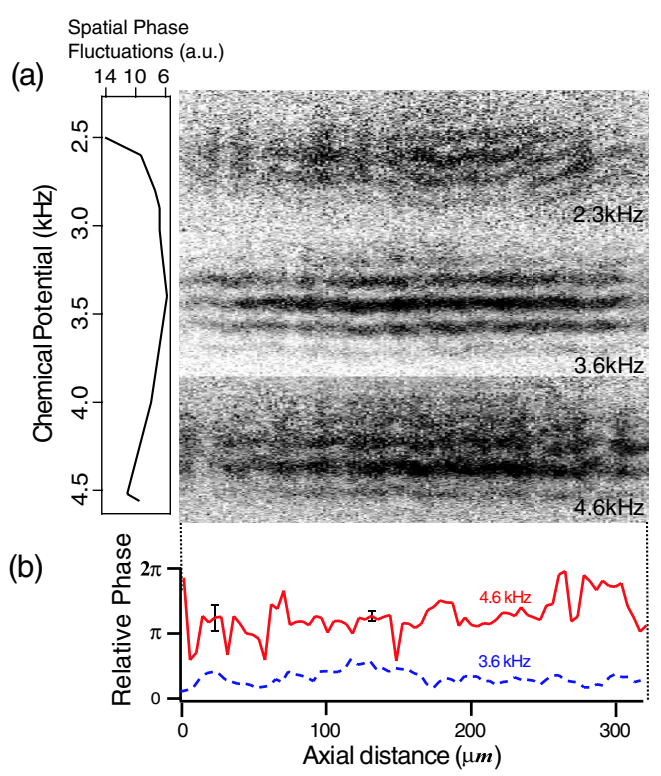

FIG. 3 (color online). Effect of spatial phase fluctuation on the waviness of interference fringes. (a) Interference fringes obtained right after splitting a condensate. For large spatial phase fluctuation (e.g., $4.6 \mathrm{kHz}$ ), the fringe pattern shows more significant wiggles than for smaller phase fluctuations (e.g., $3.6 \mathrm{kHz}$ ). (b) From the fringes for $3.6 \mathrm{kHz}$ (dashed line) and $4.6 \mathrm{kHz}$ (solid line) chemical potentials, relative phases are obtained along the axial direction. In both cases, the overall relative phase can be well determined by averaging along the axial coordinate, but considerable axial variations of the relative phase were observed in the regime of large longitudinal phase fluctuations (solid line). The error bars indicate the statistical uncertainty in the phase determination.

the splitting process, phase fluctuations would be common mode and not lead to any observable effects right after the splitting. For the smallest amount of spatial phase fluctuations, the relative phase is almost constant along the axial direction [dashed line in Fig. 3(b)]. The effect of larger phase fluctuations is displayed by the solid line. However, an average relative phase can still be determined.

To quantify the reproducibility of the relative phase, we determine the probability of the ten measurements of the relative phase being random (called randomness) [11] (Fig. 4). For values of the chemical potential larger than $3.0 \mathrm{kHz}$, the randomness is less than 0.1 which implies a reproducible phase with $90 \%$ confidence. However, by comparing Figs. 2 and 4, one clearly recognizes the degradation of reproducibility of the relative phase with increasing spatial phase fluctuations. We cannot rule out that the condensate had some weak collective excitations after preparation. However, the amount of the excitation should not depend on the final temperature. Therefore, we attribute the temperature dependence of the fringe contrast to phase fluctuations.

By introducing a variable hold time after the splitting, we can examine how spatial phase fluctuations limit the coherence time of a matter-wave interferometer. Figure 5

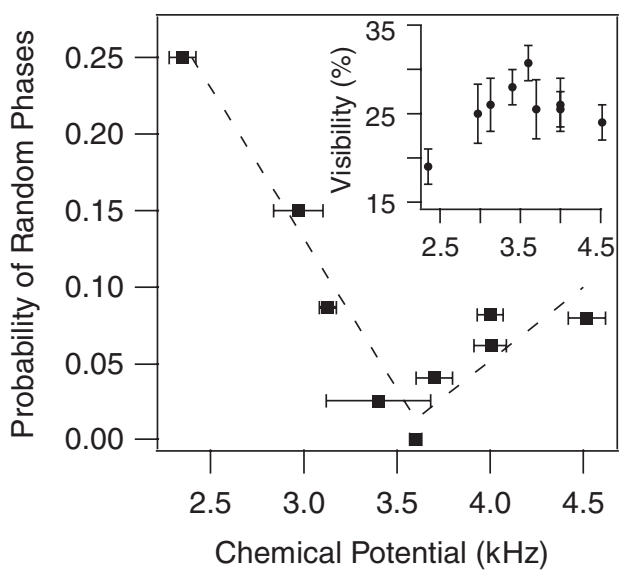

FIG. 4. Effect of spatial phase fluctuations on the reproducibility of the relative phase right after splitting. The probability of random phases was measured with variable longitudinal phase fluctuations immediately after splitting $(0 \mathrm{~ms}$ hold time $)$. The phase was determined by analyzing the central $\sim 40 \%$ of the axial length of the interference pattern. In the inset graph, the visibility of the integrated interference fringe over the central $\sim 40 \%$ is shown.

shows the increase of randomness with hold time. For the smallest amount of phase fluctuations (chemical potential $\sim 3.4 \mathrm{kHz}$, black squares in Fig. 5), the phase coherence time is $\sim 23 \mathrm{~ms}$. As the spatial phase fluctuations increase (solid circles and open squares in Fig. 5), the phase coherence time becomes shorter [22]. It should be noted that in the absence of spatial phase fluctuations, for a condensate with zero temperature, the rate of phase diffusion decreases with chemical potential, proportional to $\sim \mu^{-1 / 4}[3,4]$, which is also valid at finite temperature [3]. Our observed increase of decoherence with increasing chemical potential is therefore attributed to the increase of spatial phase fluctuations. The increasing waviness of the interference fringes show that the decoherence is caused by randomization of the relative phase along the axial direction [Fig. 5(b)].

By which mechanism do the spatial phase fluctuations affect the interferometer signal? For our experimental parameters, the rate of phase diffusion (assuming Poissonian number fluctuations after the splitting) is $\sim 20 \mathrm{~ms}[3,4]$. For our value of $T / T^{*}$, the condensate fragments into $\sim 10$ quasicondensates which should decrease the coherence time by a factor of $\sqrt{10}$ to about $7 \mathrm{~ms}$. Our observation of much longer coherence times implies strong squeezing of the relative number fluctuations, as already observed in Ref. [11]. In Ref. [11] we inferred a reduction of the number fluctuations below shot noise by a factor of 10 . However, having now established the presence of strong phase fluctuations, we should reinterprete our previous result. Those data were taken at a value of $T / T^{*}$ of about 7 , which implies that the number fluctuations for each quasicondensate was squeezed by a factor of $\sim 25$. Our current experiments were carried out in a rotated geometry (in 
(a)

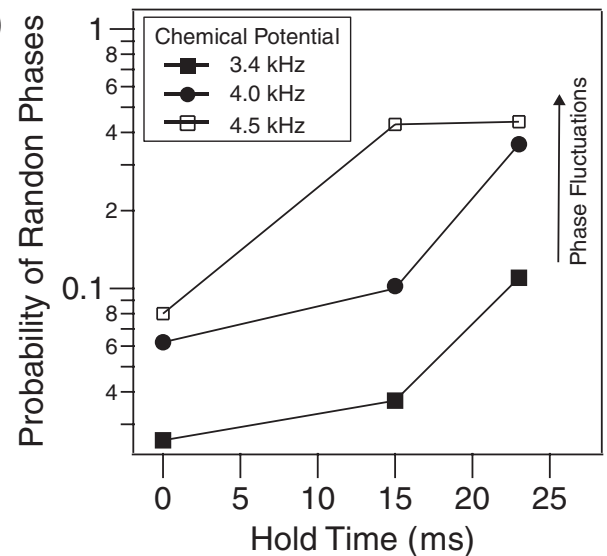

(b)

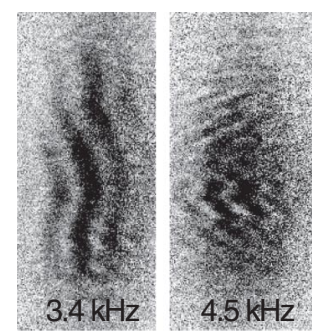

23ms Hold Time

FIG. 5. Effect of longitudinal phase fluctuations on the coherence time between the split condensates. (a) The probability for a random phase for ten measurements of the relative phase is shown for three different amounts of the longitudinal phase fluctuations. (b) For condensates in the regime of large longitudinal phase fluctuations $(\sim 4.5 \mathrm{kHz})$, interference fringes show more wavy patterns, which led to the increased randomness of the measured relative phase.

order to be able to observe along a radial direction), but the value of $T / T^{*} \sim 10$ is similar. If we assume that the squeezing factor is the same, then we should have observed phase coherence times comparable to the $200 \mathrm{~ms}$ observed previously [11].

We therefore conclude that the shorter coherence times observed in this Letter are not limited by the fundamental quantum phase diffusion of quasicondensates because of strong number squeezing, but rather reflect the interplay of spatial phase fluctuations and some relative motion of the two condensates. This is probably due to some asymmetries in the current trapping potential [23] and/or technical noise. The loss of coherence due to phase fluctuations starts already during the splitting process (Figs. 3 and 4), and increases with hold time.

The main conclusions of this Letter are that matter-wave interferometers are robust against spatial phase fluctuations, especially when strong number squeezing mitigates the fragmentation into smaller quasicondensates (which show faster phase diffusion than a single condensate) resulting in coherence times of up to $200 \mathrm{~ms}$ [11]. However, spatial phase fluctuations make the interferome- ter much more sensitive to residual relative motion of the two split condensates and therefore require a highly symmetric double-well potential.

This work was funded by DARPA, NSF, and ONR. G.-B. Jo and Y.-R. Lee acknowledge additional support from the Samsung foundation. We thank H. Kim for experimental assistance and Y. Shin for critical reading of the manuscript. We also thank E. Demler for stimulating discussions.

*http://cua.mit.edu/ketterle_group/

[1] D.S. Petrov, G. V. Shlyapnikov, and J.T.M. Walraven, Phys. Rev. Lett. 87, 050404 (2001).

[2] S. Dettmer et al., Phys. Rev. Lett. 87, 160406 (2001).

[3] M. Lewenstein and L. You, Phys. Rev. Lett. 77, 3489 (1996).

[4] Y. Castin and J. Dalibard, Phys. Rev. A 55, 4330 (1997).

[5] D. S. Petrov, M. Holzmann, and G. V. Shlyapnikov, Phys. Rev. Lett. 84, 2551 (2000).

[6] D.S. Petrov, G. V. Shlyapnikov, and J.T. M. Walraven, Phys. Rev. Lett. 85, 3745 (2000).

[7] Y. Shin et al., Phys. Rev. Lett. 92, 050405 (2004).

[8] T. Schumm et al., Nature Phys. 1, 57 (2005).

[9] S. Hofferberth et al., Nature (London) 449, 324 (2007).

[10] J. Fortagh and C. Zimmermann, Rev. Mod. Phys. 79, 235 (2007).

[11] G.-B. Jo et al., Phys. Rev. Lett. 98, 030407 (2007).

[12] G.-B. Jo et al., Phys. Rev. Lett. 98, 180401 (2007).

[13] R. Bistritzer and E. Altman, Proc. Natl. Acad. Sci. U.S.A. 104, 9955 (2007).

[14] Y. Shin et al., Phys. Rev. A 72, 021604(R) (2005).

[15] D. Hellweg et al., Phys. Rev. Lett. 91, 010406 (2003).

[16] S. Richard et al., Phys. Rev. Lett. 91, 010405 (2003).

[17] T. Gustavson, P. Bouyer, and M. Kasevich, Phys. Rev. Lett. 78, 2046 (1997).

[18] O. Zobay and B. M. Garraway, Phys. Rev. Lett. 86, 1195 (2001).

[19] S. Hofferberth et al., Nature Phys. 2, 710 (2006).

[20] For quantitative analysis of the spatial phase fluctuations, the density profile obtained from an absorption image is Fourier transformed. After filtering out high and low momentum components, the profile is transformed back and its root-mean-square average is determined (Fig. 2).

[21] In the final stage of the evaporation, the ratio of trap depth to temperature was rather small $(\approx 2)$, possibly due to heating from the atom chip potential.

[22] We did not study the temporal evolution for chemical potentials smaller than $3.4 \mathrm{kHz}$ due to the large fluctuations in the evaporation process.

[23] In our trap geometry, the two condensates in vertically separated potential wells feel different magnetic fields from a pair of end cap wires. The slightly asymmetric axial confinement leads to some quadrupolar relative motion of the two separated condensates. For the determination of the relative phase, we select the central region where the fringes were parallel to the axial direction. 